\title{
Paradoxical Motor and Cognitive Function Recovery in Response to Zolpidem in a Patient with Hypoxic-ischemic Brain Injury: A Case Report
}

\author{
Myong Hun Hahm', Jungmin $\mathbf{W o o}^{2}$ \\ Departments of ${ }^{1}$ Radiology and ${ }^{2}$ Psychiatry, School of Medicine, Kyungpook National University, Daegu, Korea
}

\begin{abstract}
We report an extremely rare case of a patient with hypoxic-ischemic brain injury who recovered consciousness and motor and cognitive functions due to paradoxical response after zolpidem administration. A 32-year-old woman who had attempted suicide by hanging was admitted. The patient had stabilized in a state of drowsy mentality, quadriparesis, dysphagia, and impaired cognition. Brain magnetic resonance imaging was suggestive of hypoxic ischemic brain injury and unilateral infarction in the right posterior cerebral artery territory. Due to sleep disturbance, zolpidem was administered, and paradoxically consciousness level and function returned to near-normal during the duration of the drug-effect. In addition to previous reports, our case characteristically showed remarkable motor and cognitive function recovery, not only consciousness level. The drug-effect time was gradually decreased after 18 months and absent after 3 years. We have reviewed related literature and discussed possible neuropharmacological and neurobiological mechanism.
\end{abstract}

KEY WORDS: Zolpidem; Brain hypoxia-ischemia; Cognition; Posterior cerebral artery infarction.

\section{INTRODUCTION}

Zolpidem is a widely used sedative drug, primarily used for the treatment of sleep disturbance. It belongs to the short-acting non-benzodiazepine class of drugs and increases the activity of gamma-aminobutyric acid (GABA), by binding to $\mathrm{GABA}_{\mathrm{A}}$ receptors at the same location as the benzodiazepines. ${ }^{1)}$ Paradoxically, however, zolpidem has been reported to restore neurologic deficits including the consciousness levels in patients with chronic consciousness disorder, including hypoxic-ischemic brain injury ( $\mathrm{HIBI})$. It is very hopeful that level of functioning which is considered as the fixed due to a broken brain can be restored by a certain drug. ${ }^{2)}$ In this context, one trial has been conducted for evaluating this promising possibility of 'awakening effects' of zolpidem." Although the result

Received: October 15, 2018 / Accepted: November 27, 2018

Address for correspondence: Jungmin Woo

Department of Psychiatry, School of Medicine, Kyungpook

National University, 680 Gukchaebosang-ro, Jung-gu, Daegu 41944, Korea

E-mail:woojm3@hanmail.net

ORCID: https://orcid.org/0000-0001-7180-9972 of this trial indicated no significant effect in group-level analysis, there were patients who show the awakening response to zolpidem. So this paradoxical phenomenon still have a meaning and we have to keep being interest in new cases which can give us understandings for finding out who can respond to zolpidem. In addition to previous reports, our patient showed remarkable change of functioning level such as motor and cognition, not only consciousness level. We report the experience of monitoring a 3-year course in an HIBI patient who showed an apparently paradoxical response to zolpidem. We have reviewed related literature and discussed possible neuropharmacological and neurobiological mechanisms.

\section{CASE}

A 32-year-old woman was referred to the emergency room. She had depressive episodes since her younger days and had attempted to commit suicide by hanging. At the time of arrival, the patient presented with comatous mentality and cardiac arrest, and cardiopulmonary cerebral resuscitation was performed. After confirming the re-

(ㄷ) This is an Open-Access article distributed under the terms of the Creative Commons Attribution Non-Commercial License (http://creativecommons.org/licenses/by-nc/4.0) which permits unrestricted non-commercial use, distribution, and reproduction in any medium, provided the original work is properly cited. 
turn of spontaneous circulation, the patient received intensive care for about 2 weeks, and an endotracheal mechanical ventilator was used for acute respiratory failure associated with aspiration pneumonia.

During hospitalization in the general ward, the patient had stabilized in a state characterized by drowsy mentality, quadriparesis, dysphagia, and impaired cognition. Usual social conversation was impossible and intermittent meaningless screaming or crying was observed. The patient showed no expressions of personal needs, did not respond accurately to simple questions, and showed verbal perseveration. The thigh muscle motor strength was grade 2/5, and other extremities were grade 3/5, hence she could not stand without help. Nasogastric tube feeding was required occasionally due to swallowing difficulty and urinary and fecal incontinence necessitated diaper use.

At 4 weeks after the suicide attempt, brain magnetic resonance imaging (MRI) was performed to evaluate the cerebral insult. Brain MRI showed bilateral symmetric diffusion restriction from the centrum semiovale to the internal capsule, anterior basal pons and pyramids of the medulla oblongata, i.e., along the corticospinal tracts, in the diffusion weighted image and apparent diffusion coefficient map (Fig. 1). These findings were consistent with Wallerian degeneration, and were indicative of a subacute stage of $\mathrm{HIBI}$, considering that the patient had a hypoxic-ischemic event 4 weeks prior. In addition, interest- ingly, a subacute stage of cerebral infarction was observed in the right posterior cerebral artery (PCA) territory (Fig. 2).

After 9 months of the suicidal hanging event, neuropsychiatric evaluations were conducted. The result of the modified Barthel index (MBI) was 7/100, which indicates 'total dependence', and those of the Mini-Mental State Examination-Korean (MMSE-K) and Global Deterioration Scale were 5/30 and stage $7 / 7$, respectively, which represent severe cognitive disorder.

As the patient showed sleep disturbance, $5 \mathrm{mg}$ of zolpidem was administered for the first time. Surprisingly and paradoxically, the patient's level of consciousness was alerted and she began to express her thoughts fluently, for example, "Why am I here?" and "Why do I look like this? I would like to groom myself." Although the patient did not recall past memories such as recognizing her parents, she communicated spontaneously, with clear pronunciation of words. Previously, she had experienced swallowing difficulty and drooling while consuming her meals, but she started to eat by herself while duration of zolpidem effect. Therefore, zolpidem was administered experimentally twice a day, morning and afternoon, and the patient exhibited near-normal activities, such as voluntary participation in exercises, washing her hands, and talking to people for about 3 hours on one dose. The score of MMSE-K rose from 5 to 16 . When zolpidem was withdrawn for experimental purposes, the patient returned to
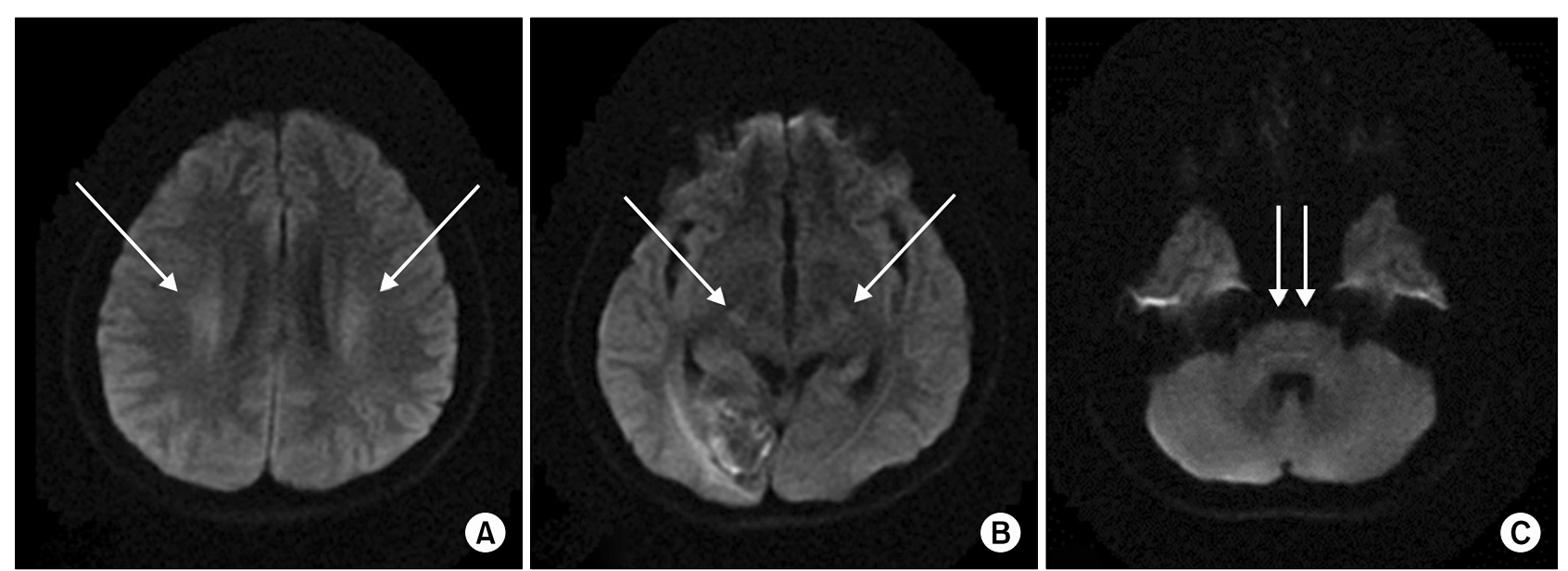

Fig. 1. Axial views of diffusion weighted image. Symmetric diffusion high signal intensity (arrows) in centrum semiovale (A), cerebral peduncles (B), and anterior basal pons (C) are observed. Lesions show iso or slightly low signal intensity in apparent diffusion coefficient map (not shown) and are not seen in other sequences. These lesions were continuously observed from the centrum semiovale to pons and matched with the pathway of the corticospinal tract. These findings indicate Wallerian degeneration, which may be seen in the subacute stage of hypoxic-ischemic brain injury. 

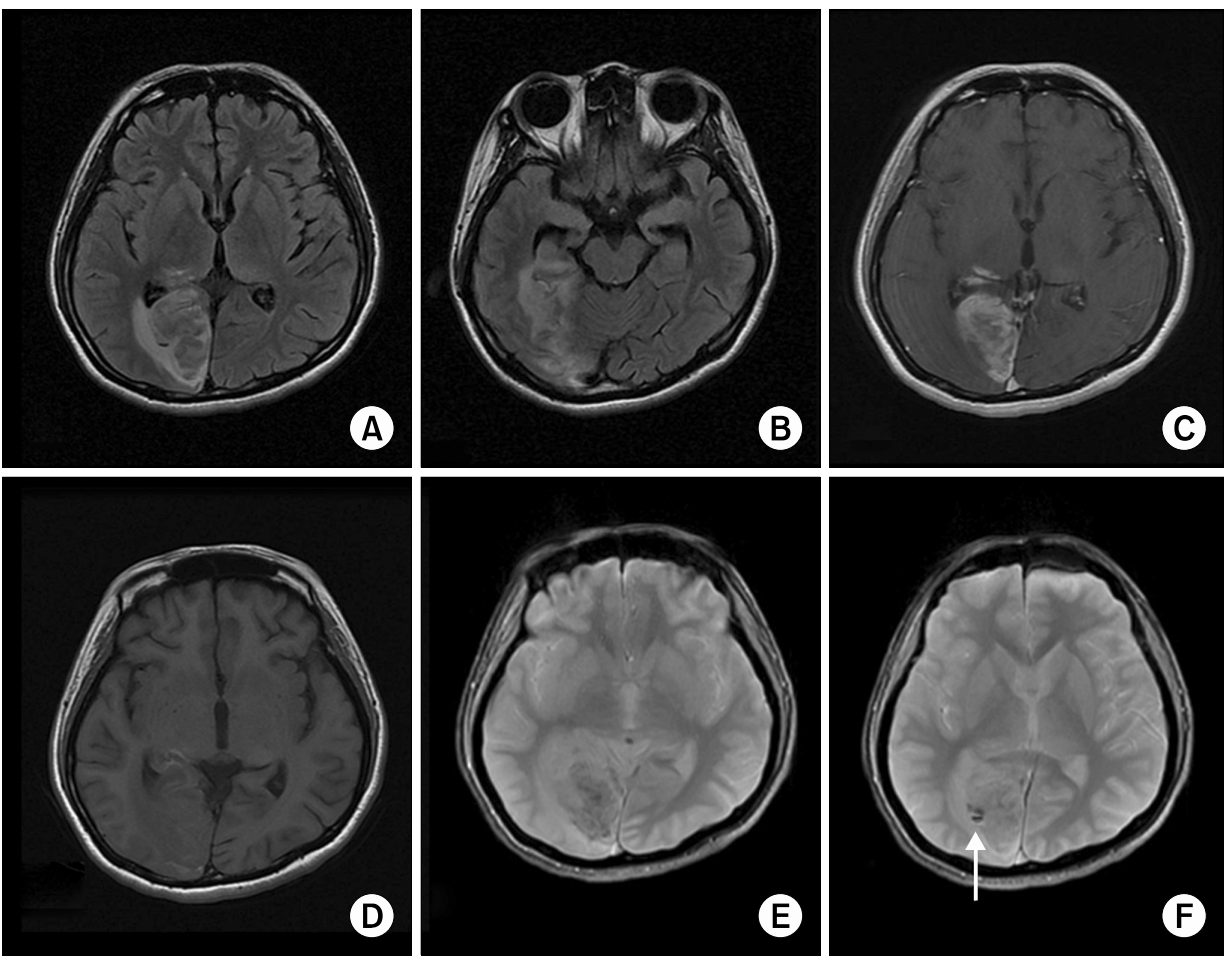

Fig. 2. Axial views of sequences showing subacute infarction in the right posterior cerebral artery (PCA) territory. High signal intensity in the right occipital lobe, mesial temporal lobe, and posterolateral thalamus on fluid-attenuated inversion recovery sequence (A, B) indicate vasogenic edema. Parenchymal enhancements in the PCA territory on gadolinium enhanced T1-weighted image (T1WI) (C), and several curvilinear high signal intensity foci on T1WI (D), which mean cortical laminar necrosis, indicate subacute stage of infarction. Dark signal intensity foci on $\mathrm{T} 2{ }^{*}$ weighted gradient echo sequence image (E) mean hemorrhagic transformation. Note the combined scanty amount of intraventricular hemorrhage (arrow in F).

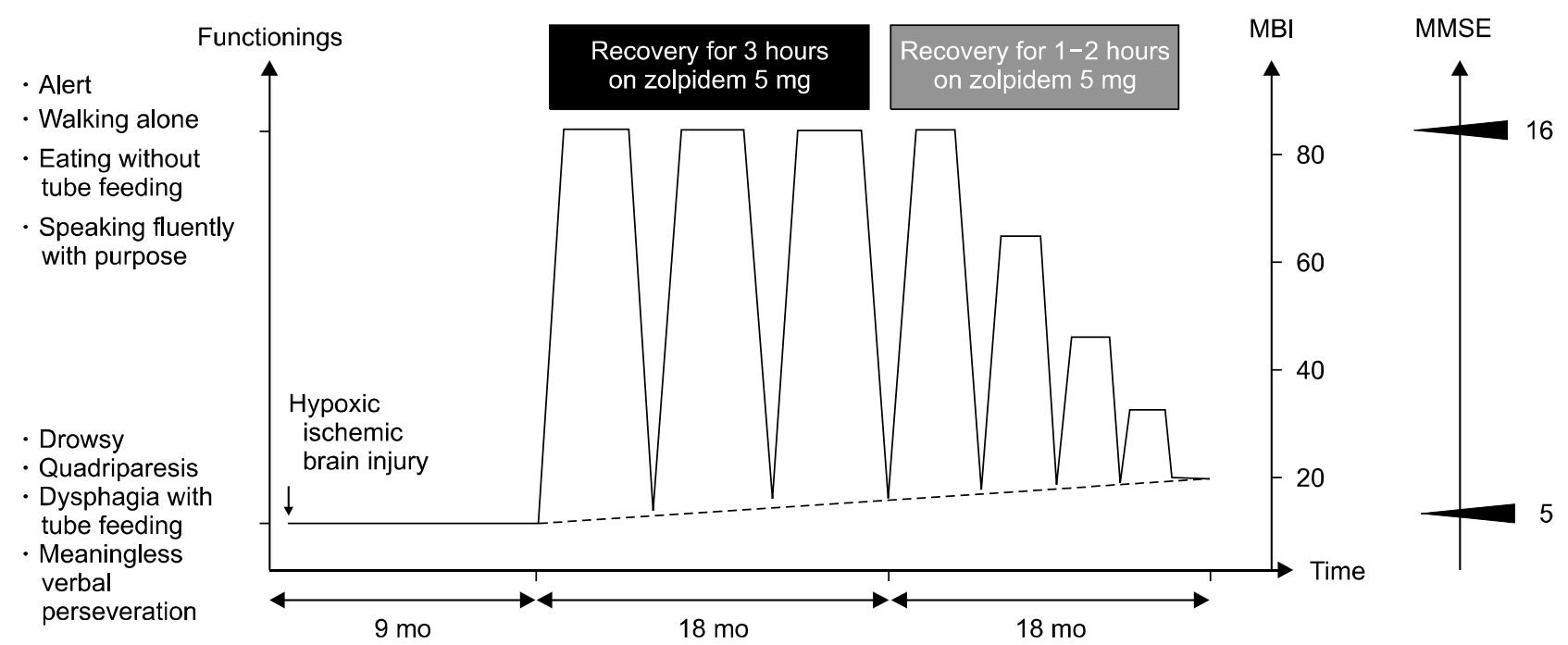

Fig. 3. The schema of the change of neuropsychiatric functional level and the modified Barthel index (MBI), Mini-Mental State Examination-Korean (MMSE-K) results by response to zolpidem over time. Note that high MBI despite sustained low MMSE-K score.

the initial basal neurological state including drowsy mentality, and $5 \mathrm{mg}$ of methylphenidate was alternatively administered, but was not effective.

However, after about 1.5 years of monitoring of the paradoxical response to zolpidem, the duration of the response began to decrease and converged to about 1 to 2 hours a day. Finally, there was no response at the third year, presumably due to drug tolerance (Fig. 3).

\section{DISCUSSION}

There have been a few reports of paradoxical response to zolpidem since 2000 that have been observed mainly in patients with brain injury; however, large prospective 
trials did not indicate significant efficacy. In other words, zolpidem appears to induce specific changes only in specific individuals with brain injury, unlike other drugs.

After brain injury, similar to the well-known hibernating myocardium, it has been proposed that the injured brain tissue changes to a dormant state. During brain hypoxia induced by any injury, limited intracellular glycogen should be used to generate glutamate, an excitatory neurotransmitter, and GABA, an inhibitory neurotransmitter. When the excitatory pathway is mainly activated, irreversible changes occur that are mediated by apoptosis and cell death. Therefore, protecting the neuron by reducing cellular metabolism, including anaerobic processes, through the inhibitory pathway by GABA is also activated. Thus, GABA receptors, mainly omega $(\omega) 1$ type $G_{A B A_{A}}$ receptors, are modified to be supersensitized by GABA so that even minimal amounts of GABA can lead to the neurodormancy pathway. ${ }^{1,4)}$

Zolpidem is a high-affinity positive modulator of the $\omega 1$ type $\mathrm{GABA}_{\mathrm{A}}$ receptors and selectively binds to the alpha 1 subunit of the receptor in contrast to benzodiazepine, which is a non-selective ligand. Zolpidem modifies the receptor by inducing a receptor conformation. ${ }^{5,6)}$ Therefore, it is presumed that zolpidem binds specifically to the supersensitized modified $\omega 1$ type $G A B A_{A}$ receptors and distorts the receptor structure to release it from the neurodormant state. Therefore, it can be thought that zolpidem may show a dramatic clinical effect in those particular individuals with numerous neurodormant cells or those in which neurodormant cells dominate large regions of the brain. ${ }^{1,4,7)}$

The patient had a strong risk factor for $\mathrm{HIBI}$, including cardiac arrest and acute respiratory failure. MRI revealed Wallerian degeneration suggesting subacute phase $\mathrm{HIBI}^{8}{ }^{8,9)}$ However, except for the right PCA territory, there was a small overall cortical neuronal loss. Unilateral PCA territory infarction may have some influences on consciousness level or cognitive function; however, the motor function deficit was difficult to explain. Therefore, we can conclude that there was diffuse neuronal damage that could not be detected in the MRI, or that the neurons were rendered dormant. Accordingly, we can assume that a paradoxical response may occur when a large proportion of dormant neurons are 'switched on' by zolpidem, only in cases of small neuronal loss, as in our patient.

Considering a different point of view, it is also rare that unilateral cerebral infarction, especially PCA territorial infarction, is observed in a 'nearly-hung' individual. It is generally believed that localized infarction may occur with a clot due to direct injury or compression of internal cerebral artery or vertebral artery during hanging; however, this is usually reported to occur bilaterally. Some reports suggest that unilateral cerebral infarction may occur in hanging events within 5 minutes. Therefore, this finding also suggests that our patients had shorter ischemic time and may have HIBI with a small neuronal loss. ${ }^{10)}$ Our patient showed a high MBI, that is high functioning level, due to the paradoxical response to zolpidem, despite sustained low MMSE score (Fig. 3). This also suggests the possibility of small neuronal loss in our patient and zolpidem may help to restore functioning levels in patients with low MMSE score but low neuronal loss.

Conclusively, we expect that our case may provide some clues to understand the micro-environmental changes in $\mathrm{HIBI}$ and the hypotheses regarding the short- and long-term paradoxical effects of zolpidem in HIBI patients with small neuronal loss will require precisely controlled trials. Furthermore, if we can develop an alternative drug with a less tolerance in patients, it can help improve the functioning in HIBI patients.

\section{- Conflicts of Interest}

No potential conflict of interest relevant to this article was reported.

\section{REFERENCES}

1. Kim C, Kwon BS, Nam KY, Park JW, Lee HJ. Zolpidem-induced arousal by paradoxical GABAergic stimulation: a case report with F-18 flumazenil positron emission tomography and single photon emission computed tomography study. Ann Rehabil Med 2016;40:177-181.

2. Lee JO, Kim JW, Kang HJ, Hong JP, Kim JM. Predictors of cognitive improvement during 12 weeks of antidepressant treatment in patients with major depressive disorder. Clin Psychopharmacol Neurosci 2018;16:461-468.

3. Thonnard M, Gosseries O, Demertzi A, Lugo Z, Vanhaudenhuyse A, Bruno MA, et al. Effect of zolpidem in chronic disorders of consciousness: a prospective open-label study. Funct Neurol 2013;28:259-264.

4. Clauss R, Nel W. Drug induced arousal from the permanent vegetative state. NeuroRehabilitation 2006;21:23-28.

5. Rowlett JK, Woolverton WL. Assessment of benzodiazepine receptor heterogeneity in vivo: apparent $p A 2$ and $p K B$ analyses from behavioral studies. Psychopharmacology (Berl) 
1996; 128:1-16.

6. Perrais D, Ropert N. Effect of zolpidem on miniature IPSCS and occupancy of postsynaptic GABAA receptors in central synapses. J Neurosci 1999;19:578-588.

7. Williams ST, Conte MM, Goldfine AM, Noirhomme Q, Gosseries $\mathrm{O}$, Thonnard $\mathrm{M}$, et al. Common resting brain $d y$ namics indicate a possible mechanism underlying zolpidem response in severe brain injury. Elife 2013;2:e01157.

8. Mazumdar A, Mukherjee P, Miller JH, Malde H, McKinstry RC. Diffusion-weighted imaging of acute corticospinal tract injury preceding Wallerian degeneration in the maturing human brain. A/NR Am J Neuroradiol 2003;24:1057-1066.

9. Hald JK, Brunberg JA, Dublin AB, Wootton-Gorges SL. Delayed diffusion-weighted MR abnormality in a patient with an extensive acute cerebral hypoxic injury. Acta Radiol 2003; 44:343-346.

10. Garaci FG, Bazzocchi G, Velari L, Gaudiello F, Goldstein AL, Manenti G, et al. Cryptogenic stroke in hanging. A case report. Neuroradiol J 2009;22:386-390. 